\title{
Alanine Aminotransferase and 20-Year Risk of Major Chronic Diseases and Death in a Healthy Cohort Aged 30 to 49 Years
}

This article was published in the following Dove Press journal: Clinical Epidemiology

\author{
Morten Daniel Jensen (1D' \\ Torsten Lauritzen ${ }^{2}$ \\ Hendrik Vilstrup' \\ Peter Jepsen $\mathbb{D}^{1,3}$
}

'Department of Hepatology and Gastroenterology, Aarhus University Hospital, Aarhus, Denmark; ${ }^{2}$ Department of Public Health, Research Group for General Practice, University of Aarhus, Aarhus, Denmark; ${ }^{3}$ Department of Clinical Epidemiology, Aarhus University Hospital, Aarhus, Denmark
Correspondence: Morten Daniel Jensen Department of Hepatology and Gastroenterology, Aarhus University Hospital, Entrance C, Plan I, Intersection CII6, Palle Juul-Jensens Boulevard 99, Aarhus N 8200, Denmark

Tel +45 27597549

Email moje@clin.au.dk
Purpose: Alanine aminotransferase is the most frequently used marker of liver cell injury. We examined the association between alanine aminotransferase levels and long-term absolute risks of morbidity and mortality in healthy Danish people aged 30-49 years.

Patients and Methods: We divided 671 healthy participants from the Ebeltoft Health Promotion Project into four categories based on their baseline alanine aminotransferase values: low ( $\leq 10 \mathrm{U} / 1)$, medium-low (men: 11-34U/1, women: 11-22U/1), medium-high (men: 35-69U/1, women: 23-44U/1) and high (men: $\geq 70 \mathrm{U} / 1$, women: $\geq 45 \mathrm{U} / 1$ ), and followed them through Danish healthcare registries for up to 20 years. We examined mortality and absolute risks of liver disease, overall cancer, ischemic heart disease, and diabetes.

Results: The risk of any cancer was highest for participants with "low alanine aminotransferase" or "high alanine aminotransferase" (20-year risk: 17.2\% [95\% confidence interval (CI): $6.3-32.7 \%$ ] and 18.2\% [95\% CI: 5.7-36.3\%], respectively). The risk of diabetes was highest for participants with "medium-high alanine aminotransferase" or "high alanine aminotransferase" (20-year risk: 12.1\% [95\% CI: 7.3-18.3\%] and 9.1\% [95\% CI: $1.6-$ 25.1\%], respectively). Participants with "high alanine aminotransferase" had the highest 20year risk of liver disease (20-year risk: $13.6 \%$ [95\% CI: $3.4-30.9 \%$ ], while it was $1.0 \%$ or less in the other groups). The chance of being alive after 20 years without having been diagnosed with liver disease, cancer, ischemic heart disease, or diabetes was lowest in the "high alanine aminotransferase" group (50\% [95\% CI: 28-68\%]) and 72-79\% in the other groups.

Conclusion: Our findings suggest that persons with high or abnormally low alanine aminotransferase measurements are at increased long-term risk of several chronic diseases.

Keywords: ALT, mortality, morbidity, cohort study

\section{Plain Language Summary}

Alanine aminotransferase is an enzyme predominantly found in the liver cells, and the blood concentration of alanine aminotransferase is the most frequently used marker of liver injury. We examined risks of death and selected chronic diseases during 20 years of follow-up among healthy members of the Danish general population aged 30-49 years who participated in the Ebeltoft Health Promotion Project. These persons' alanine aminotransferase concentration was measured at their general practitioner in connection with a health check-up. We found that those with an abnormal low alanine aminotransferase concentration were at high risk of cancer (cumulative risk of $17.2 \%$ after 20 years), and that those with an abnormal high alanine aminotransferase concentration were at high risk of liver disease $(13.6 \%$ after 20 years), cancer (18.2\% after 20 years), ischemic heart disease (9.1\% after 20 years), and 
diabetes (9.1\% after 20 years). These findings suggest that persons with a very low or a very high alanine aminotransferase concentration are at high long-term risk of several chronic diseases.

\section{Introduction}

Alanine aminotransferase (ALT) is an enzyme predominantly found in the liver. The plasma ALT level is the most frequently used marker of liver cell injury. ${ }^{1,2}$ Earlier studies have examined the association between plasma ALT concentration and the relative risks of mortality, diabetes and cardiovascular disease, but results have been contradictory, ${ }^{3-6}$ and still the long-term absolute risks of morbidity and mortality in healthy people remain unknown. We studied a healthy general population sample of persons aged 30-49 years to examine the association between baseline plasma ALT concentration and 20-year risk of liver disease, overall cancer, ischemic heart disease, diabetes, and death.

\section{Materials and Methods}

\section{Data Sources}

\section{Ebeltoft Health Promotion Project}

The Ebeltoft Health Promotion Project was a randomized controlled trial designed to examine the value of health check-ups in the general population. ${ }^{7-9}$ All trial participants were 30-49 years old on 1 January 1991 and lived in the Danish town of Ebeltoft (total population $=13,000$ persons). Among the 3464 trial participants, 1006 were randomized to undergo one or more health check-ups. Of these, 905 participants had a baseline health check-up, carried out between December 1991 and June 1992, and 671 of these participants met the inclusion criteria of our study. The check-up included a questionnaire about lifestyle factors and a blood sample for analysis of serum biochemistry. Here we examine the long-term prognosis of these 671 trial participants.

\section{Danish Healthcare Registries}

We followed the 671 participants through the year 2012 using several Danish healthcare registries. ${ }^{10}$ The Danish Pathology Data Bank contains data from all histological examinations, coded according to the SNOMED classification. This Pathology Data Bank became nationwide in 1997, but most pathology departments have contributed data from earlier years. ${ }^{11}$ The Danish National Patient Register contains individual-level data on all Danish patients receiving hospital inpatient and outpatient treatment, with hospital discharge diagnoses coded according to the International Classification of Diseases, Eighth Revision (ICD-8) during 1977-1993 and according to the Tenth Revision (ICD-10) since 1994. ${ }^{12}$ The Danish Cancer Registry contains individual-level data on cancers diagnosed in Denmark, coded according to the ICD for oncology; this Registry dates back to $1943 .{ }^{13}$ The Danish Register of Causes of Death contains data on causes of death for all Danish decedents. ${ }^{14}$ The Danish Civil Registration System issues the unique personal identification number that is given to all Danish residents at birth or upon immigration. This database records dates of birth, death, and migration for all Danish residents. ${ }^{15}$

Individual-level data from these Danish healthcare registries and the Ebeltoft Health Promotion Project were linked using personal identification numbers.

\section{Definition of Disease and Follow-Up}

Participants were followed from their baseline health checkup until they died or were diagnosed with liver disease, cancer, ischemic heart disease, or diabetes, whichever came first (diagnosis codes shown in Supplementary material). We chose liver disease, cancer, ischemic heart disease, and diabetes as end points; cancer and ischemic heart disease because they are the two most common causes of death in Denmark, ${ }^{16}$ liver disease and diabetes because they are likely to be associated with high ALT levels. Participants who had no outcome events before 1 January 2013 were censored on that date. Only three participants were lost to follow-up; they emigrated and were censored on the date of emigration.

\section{Statistical Analysis}

We categorized participants into four categories based on their plasma ALT at baseline: low (ALT $\leq 10 \mathrm{U} / 1)$, mediumlow (ALT 11-34 U/1 for men, 11-22 U/1 for women), medium-high (ALT 35-69 for men, 23-44 for women) and high ( $\geq 70 \mathrm{U} / 1$ for men, $\geq 45 \mathrm{U} / 1$ for women). The ALT categories were based on the reference intervals used in clinical practice in Denmark, which are derived from "The Nordic Reference Interval Project". ${ }^{17}$ The low and high ALT reference limits were the 2.5- and 97.5 percentile of that study, so we expect the vast majority of participants to be in the medium-low or medium-high groups. We calculated the absolute risks of liver disease, any cancer, ischemic heart disease, and diabetes for participants based on their ALT categorization by means of the cumulative incidence function, ${ }^{18}$ where these diseases and "death" (specifically, 'death without liver disease, cancer, ischemic heart disease, or diabetes') were treated as competing risks. 
To examine all-cause mortality, we computed cumulative mortality using the Kaplan-Meier method; hence we did not consider any competing events in that analysis.

Lastly, we did a Cox proportional hazards regression, with ALT as a continuous variable and fitted using penalized splines, ${ }^{19}$ while adjusting for sex, age, and smoking status. The modelling purpose was to examine the effect of liver cell injury of whichever cause on the rates of liver disease, overall cancer, ischemic heart disease, diabetes, and all-cause mortality while allowing for the possibility that those associations might not be linear. The degrees of freedom for the splines were chosen so that the corrected Akaike information criterion (AICc) was minimized. ${ }^{20}$

\section{Results}

Of the 905 participants initially identified, 24 had received a diagnosis of liver disease, cancer, ischemic heart disease or diabetes before inclusion, and further 210 reported having a chronic disease in their questionnaire and were excluded from the analyses. None were excluded based on their ALT measurement. This left 671 participants in our analyses, in the following four groups: low (29 persons), medium-low (488 persons), medium-high (132 persons) and high ALT (22 persons). The 671 participants had a median follow-up time of 20 years and a total follow- up time of 12,082 years. The proportion of males overall and within 3 of the 4 ALT groups was close to $50 \%$, but the low ALT group was only 3.4\% male. The median age at inclusion was close to 40 years in all groups. Persons with a higher body mass index had higher ALT concentrations. The median number of alcohol units per week was 6 , also increasing with higher ALT values. We found no association between ALT concentration and marital status or smoking status, except that the small group with high ALT had many current smokers (Table 1).

The absolute risk of liver disease was much higher for people with high ALT concentrations than for those in the other groups, who had similar risks. Persons with extreme ALT concentrations, whether high or low, had a higher overall absolute cancer risk than other persons (20-year risk: 18.2\% [CI: 5.7-36.3] for high ALT and 17.2\% [CI: 6.3-32.7] for low ALT) (cancer types shown in Table S1). There was no apparent association between ALT and absolute risk of ischemic heart disease. The absolute risk of diabetes increased with increasing ALT concentration (Table 2).

Absolute mortality risk was highest in the extreme ALT groups (Figure 1), slightly more so in the low ALT group than in the high ALT group (20-year risk, respectively: $13.8 \%$ [95\% CI: $5.4-32.7 \%$ ] and $9.1 \%$ [95\% CI: $2.4-$ $31.7 \%$ ] and risk ratio $13.8 / 9.1=1.5$ [95\% CI: $0.3-7.5]$ ).

Table I Baseline Characteristics of 67I Healthy Participants Ages 30-49 from the Ebeltoft Health Promotion Project in Denmark, I99|-1992

\begin{tabular}{|c|c|c|c|c|c|}
\hline ALT & $\begin{array}{l}\text { Low } \\
(N=29)\end{array}$ & $\begin{array}{l}\text { Medium-Low } \\
(\mathbf{N}=488)\end{array}$ & $\begin{array}{l}\text { Medium-High } \\
(\mathbf{N}=132)\end{array}$ & $\begin{array}{l}\text { High } \\
(N=22)\end{array}$ & $\begin{array}{l}\text { Total } \\
(N=67 I)\end{array}$ \\
\hline \multicolumn{6}{|l|}{ Sex, (\%) } \\
\hline Male & I (3.4) & $237(48.6)$ & $76(57.6)$ & $14(63.6)$ & $328(48.9)$ \\
\hline Female & $28(96.6)$ & $25 \mathrm{I}(5 \mathrm{I} .4)$ & $56(42.4)$ & $8(36.4)$ & $343(5 \mathrm{I} . \mathrm{I})$ \\
\hline Age, median (IQR) & $39.0(35.0-43.0)$ & $41.0(36.0-45.0)$ & $39.5(34.5-45.0)$ & $42.5(39.0-46.0)$ & $40.0(36.0-45.0)$ \\
\hline Weight ${ }^{\mathrm{a}}$, median (IQR) & $61.0(57.0-66.5)$ & $70.0(60.0-80.0)$ & $82.0(70.0-90.0)$ & $73.0(60.0-85.0)$ & $71.5(61.0-82.0)$ \\
\hline $\mathrm{BMI}^{\mathrm{b}}$, median (IQR) & $21.5(20.7-24.2)$ & $23.3(2 \mathrm{I} .4-25.7)$ & $25.9(24.0-28.7)$ & $24.4(21.7-27.8)$ & $23.8(21.6-26.5)$ \\
\hline \multicolumn{6}{|l|}{ Smoking Status, (\%) } \\
\hline Never & II (37.9) & $177(36.3)$ & $51(38.6)$ & $3(13.6)$ & $242(36.1)$ \\
\hline Former & $5(17.2)$ & $61(12.5)$ & $17(12.9)$ & $5(22.7)$ & $88(13.1)$ \\
\hline Current & $13(44.8)$ & $250(5 \mathrm{I} .2)$ & $64(48.5)$ & $14(63.6)$ & $34 I(50.8)$ \\
\hline Weekly alcohol consumption ${ }^{c}$, median (IQR) & $3(2-7)$ & $6(2-13)$ & $7(2-20)$ & $19(10-28)$ & $6(2-14)$ \\
\hline \multicolumn{6}{|l|}{ Marital Status ${ }^{\mathrm{d}},(\%)$} \\
\hline Married or living together & $24(82.8)$ & $420(86.2)$ & II 2 (84.9) & $17(77.3)$ & $573(85.5)$ \\
\hline Living alone & $5(17.2)$ & $67(13.8)$ & $20(15.2)$ & $5(22.7)$ & 97 (I4.5) \\
\hline
\end{tabular}

Notes: ${ }^{a}$ Measured in kg; Data was missing for 13 participants (Medium-Low: 10, Medium-High: 3) bata was missing for 22 participants (Medium-Low: 16, Medium-High: 6) 'Measured in units of $\mathrm{I} 2 \mathrm{~g}$ alcohol/unit; data was missing for 12 participants (Low: I, Medium-Low: 8, Medium-High: 3 ) ${ }^{\mathrm{d} D a t a}$ was missing for I participant (Medium-Low: I). Abbreviations: IQR, interquartile range (ie 25th-75th percentile); BMI, body mass index $\left(\mathrm{kg} / \mathrm{m}^{2}\right)$. 
Table 2 Cumulative Incidence by ALT Category and Outcome Event

\begin{tabular}{|c|c|c|c|c|}
\hline & \multicolumn{4}{|c|}{ Cumulative Incidence (\%) (95\% Confidence Interval) } \\
\hline & Low $(\mathbf{N}=29)$ & Medium-Low $(\mathrm{N}=488)$ & Medium-High $(\mathbf{N}=132)$ & High $(\mathbf{N}=22)$ \\
\hline \multicolumn{5}{|l|}{ Liver Disease } \\
\hline 10-year risk & 0 & $0.6(0.2-1.7)$ & $0.8(0.1-3.8)$ & $4.5(0.3-18.9)$ \\
\hline 20-year risk & 0 & $1.0(0.4-2.3)$ & $0.8(0.1-3.8)$ & $13.6(3.4-30.9)$ \\
\hline \multicolumn{5}{|l|}{ Overall Cancer } \\
\hline 10-year risk & $10.3(2.6-24.3)$ & $3.1(1.8-4.9)$ & $0.8(0.1-3.8)$ & $4.5(0.3-18.9)$ \\
\hline 20-year risk & $17.2(6.3-32.7)$ & $8.4(6.2-11.1)$ & $6.1(2.8-11.0)$ & $18.2(5.7-36.3)$ \\
\hline \multicolumn{5}{|l|}{ Ischemic Heart Disease } \\
\hline 10-year risk & $3.4(0.3-14.9)$ & $3.7(2.3-5.6)$ & $0.8(0.1-3.8)$ & 0 \\
\hline 20-year risk & $3.4(0.3-14.9)$ & $9.3(6.9-12.0)$ & $6.8(3.3-12.0)$ & $9.1(1.6-25.1)$ \\
\hline \multicolumn{5}{|l|}{ Diabetes } \\
\hline 10-year risk & 0 & $0.4(0.1-1.4)$ & 4.5 (1.9-9.1) & $4.5(0.3-18.9)$ \\
\hline 20-year risk & $3.4(0.3-14.9)$ & $1.0(0.4-2.3)$ & $12.1(7.3-18.3)$ & $9.1(1.6-25.1)$ \\
\hline \multicolumn{5}{|l|}{ No Event } \\
\hline 10-year risk & 86 (67-95) & 92 (89-94) & $92(85-95)$ & $86(63-95)$ \\
\hline 20-year risk & $76(56-88)$ & 79 (75-82) & $72(63-79)$ & $50(28-68)$ \\
\hline \multicolumn{5}{|l|}{ Any Event } \\
\hline 10-year risk & $14(5.4-33)$ & $8.2(6.1-11)$ & $8.3(4.7-15)$ & $14(4.6-37)$ \\
\hline 20-year risk & $24(12-44)$ & $2 \mid(\mid 8-25)$ & $28(2 \mid-37)$ & $50(32-72)$ \\
\hline
\end{tabular}

Notes: Note that the risk of "any event" is the sum of the risks of a diagnosis of liver disease, overall cancer, ischemic heart disease, or diabetes, as well as 'death without liver disease, cancer, ischemic heart disease, or diabetes' (before rounding). It is also $100 \%$ minus the "risk" of no event.

After 20 years of follow-up, participants with a low or medium ALT concentration had a $72 \%$ to $79 \%$ chance of being alive without having been diagnosed with liver disease, cancer, ischemic heart disease, or diabetes. The corresponding probability for persons with a high ALT was only 50\% (95\% CI: 28-68\%) (Table 2).

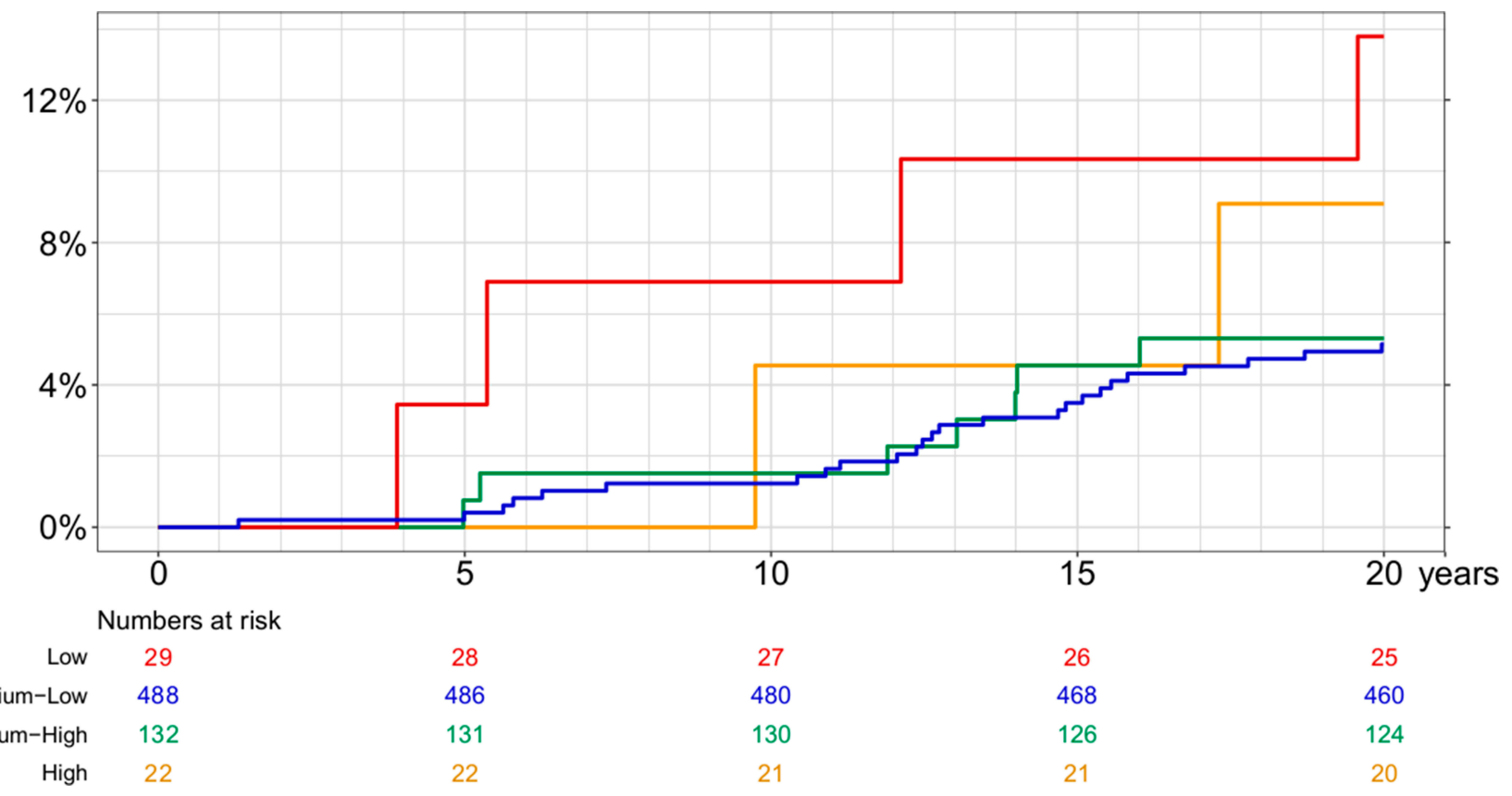

Figure I Cumulative all-cause mortality by ALT concentration. 

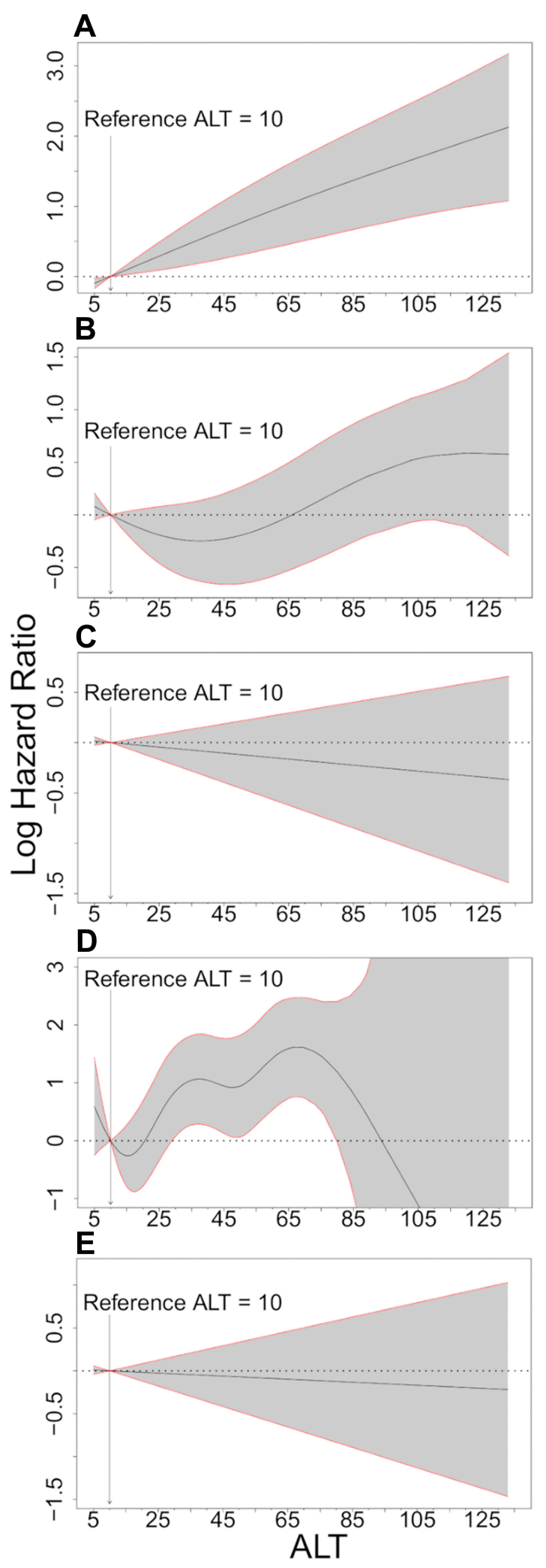

Figure 2 Hazard ratios of liver disease, overall cancer, ischemic heart disease, diabetes, and all-cause mortality adjusted for sex, age, and smoking status. (A) Liver Disease (B) Overall cancer (C) Ischemic Heart Disease (D) Diabetes (E) All-Cause Mortality.
When we used splines to allow flexibility in the associations between ALT as a continuous variable and disease rates and adjusted for differences in gender, age, and smoking status, we found roughly the same associations as indicated by Table 2 . Thus, the rate of liver disease development increased with ALT level, the rate of cancer development was increased for all patients with an abnormal ALT, the rate of ischemic heart disease showed no apparent association with ALT levels, and the rate of diabetes development increased with increasing ALT but was very imprecisely estimated at high ALT levels. Allcause mortality was only weakly associated with ALT (Figure 2).

\section{Discussion}

Healthy people who present to their general practitioner for a health check-up at age 30-49 deserve accurate information about the implications of abnormal findings. We found that those with an abnormally high ALT concentration had the worst prognosis, with a $14 \% 20$-year risk of liver disease and just a $50 \%$ chance of not dying or developing liver disease, cancer, ischemic heart disease, or diabetes within 20 years. The prognosis was better for people with medium or low ALT values, but it was notable that those with a medium-high ALT had a high risk of diabetes and those with an abnormally low ALT had a high risk of cancer.

We studied a representative sample of the general Danish population, ${ }^{21}$ and found that liver cell injury was associated with high alcohol consumption, smoking, and possibly an overall unhealthier lifestyle. We could not disentangle the effect of liver cell injury from the effects of the underlying causes of that liver cell injury, most notably alcohol, overweight, existing liver disease, medications, and/or genetic susceptibility to liver injury. The participants were not screened for hepatitis B or hepatitis $\mathrm{C}$, however, the prevalence in Denmark is only $0.24 \%$ and $0.38 \%$, respectively, making it unlikely that a high ALT is due to unidentified hepatitis B or C. ${ }^{22,23}$ We excluded participants with existing liver disease, but we chose not to adjust for alcohol intake and overweight because that would not help the interpretation. We believe that any causal effect of a minor liver injury on the development of chronic diseases up to 20 years later is far smaller than the causal effects of whichever factors caused that minor liver injury. Having repeated ALT measurements or mea- 
surements of other liver enzymes could have helped in understanding the causal effect of liver cell injury and therefore we see the liver cell injury, represented by the ALT level, as predictive rather than causal.

The high risk of diabetes among participants with a high ALT and the lack of an association between ALT and ischemic heart disease is consistent with other studies. ${ }^{4}$ Other studies have found that a low ALT is associated with loss of independence and death, ${ }^{24}$ and a marker of low muscle mass due to malnutrition and/or cancer. Those findings could help explain the associations with cancer and all-cause mortality for low ALT in our study. However, our sample size in the low ALT group was small and the estimates were not significantly different from the other groups. ${ }^{3}$ If we could follow participants for longer, we believe that also a high ALT would be associated with increased mortality because of its association with development of liver disease and diabetes.

Our findings suggest that a healthy person aged 30-49 years who presents with a low, medium-high, or high ALT measurement might benefit from a follow-up visit to his or her general practitioner focusing on modifiable risks. For persons with a low ALT value that visit might focus on cancer risk factors. For persons with a medium-high ALT value the visit should focus on diabetes risk factors, and for persons with a high ALT value the focus should be on risks factors for cancer, diabetes, and liver disease. The participants we studied were offered follow-up health checks, but those visits did not affect the risks of cardiovascular disease or death. ${ }^{21}$

\section{Conclusions}

In conclusion, our study is the first to assess the long-term absolute risk of morbidity and mortality related to measurements of ALT in healthy people who visited a general practitioner for a health check-up. We found that participants with abnormal high or low ALT levels were at increased risk of several chronic diseases.

\section{Abbreviations}

ALT, Alanine aminotransferase; ICD, International Classification of Diseases; CI, Confidence interval.

\section{Data Sharing Statement}

Data are available upon reasonable request.

\section{Ethics and Consent Statement}

This project, where data from an ethical committeeapproved project is linked to data from Danish healthcare registries, does not require a new approval from an ethics committee, according to Danish law.

\section{Disclosure}

The abstract of this paper was presented at the "The International Liver Congress 2019" as a poster presentation with interim findings. The poster's abstract was published in "Poster Presentations" in "Journal of Hepatology": https:// doi.org/10.1016/S0618-8278(19)30648-6.

The authors report no conflicts of interest in this work.

\section{References}

1. Charles Patrick Davis M. Liver blood tests (Normal, Low, and High Ranges \& Results). 2017. Available from: https://www.medicinenet. com/liver_blood_tests/article.htm\#what_are_the_basic_functions_of the liver. Accessed January 30, 2020.

2. Ozer J, Ratner M, Shaw M, Bailey W, Schomaker S. The current state of serum biomarkers of hepatotoxicity. Toxicology. 2008;245 (3):194-205. doi:10.1016/j.tox.2007.11.021

3. Ruhl CE, Everhart JE. The association of low serum alanine aminotransferase activity with mortality in the US population. $\mathrm{Am}$ $J$ Epidemiol. 2013;178(12):1702-1711. doi:10.1093/aje/kwt209

4. Goessling W, Massaro JM, Vasan RS, D'agostino RB, Ellison RC, Fox CS. Aminotransferase levels and 20-year risk of metabolic syndrome, diabetes, and cardiovascular disease. Gastroenterology. 2008;135(6):1935-1944.e1931. doi:10.1053/j.gastro.2008.09.018

5. Yuwaki K, Shimazu T, Yamagiwa Y, et al. Association between serum liver enzymes and all-cause mortality: the Japan Public Health Center based Prospective Study. Liver Int. 2019;39 (8):1566-1576. doi:10.1111/liv.v39.8

6. Unalp-arida A, Ruhl CE. Noninvasive fatty liver markers predict liver disease mortality in the U.S. population. Hepatology. 2016;63 (4):1170-1183. doi:10.1002/hep.28390

7. Susanne Reindahl Rasmussen JK, Hvenegaard A, Thomsen JL, Engberg M, Lauritzen T, Søgaard J. Forebyggende helbredsundersøgelser og helbredssamtaler i almen praksis - En sundhedsøkonomisk analyse af »Sundhedsprojekt Ebeltoft«. Available f r o m: h t t p : / / w w w. s s t. d k / / m e d i a / BEBA7098FBAA4637A7D10BB30215A404.ashx. Accessed January 30, 2020.

8. Lauritzen T, Leboeuf-yde C, Lunde IM, Nielsen KD. Ebeltoft project: baseline data from a five-year randomized, controlled, prospective health promotion study in a Danish population. Br J Gen Pract. 1995;45(399):542-547.

9. Lauritzen T, Jensen MS, Thomsen JL, Christensen B, Engberg M. Health tests and health consultations reduced cardiovascular risk without psychological strain, increased healthcare utilization or increased costs: an overview of the results from a 5-year randomized trial in primary care. The Ebeltoft Health Promotion Project (EHPP). Scand J Public Health. 2008;36(6):650-661. doi:10.1177/1403494807090165

10. Schmidt M, Schmidt SAJ, Sundbøll KAJ, Ehrenstein KLV, Sørensen HT. The Danish health care system and epidemiological research: from health care contacts to database records. Clin Epidemiol. 2019; Volume 11. doi:10.2147/CLEP

11. Bjerregaard B, Larsen OB. The Danish pathology register. Scand J Public Health. 2011;39(7 Suppl):72-74. doi:10.1177/1403494810 393563 
12. Lynge E, Sandegaard JL, Rebolj M. The Danish national patient register. Scand J Public Health. 2011;39(7 Suppl):30-33. doi:10.11 77/1403494811401482

13. Gjerstorff ML. The Danish cancer registry. Scand J Public Health. 2011;39(7 Suppl):42-45. doi:10.1177/1403494810393562

14. Helweg-larsen K. The Danish register of causes of death. Scand J Public Health. 2011;39(7 Suppl):26-29. doi:10.1177/1403494811399958

15. Pedersen CB. The Danish civil registration system. Scand J Public Health. 2011;39(7 Suppl):22-25. doi:10.1177/1403494810387965

16. Sundhedsdatastyrelsen. Dødsårsagsregistret 2018 - Tal og analyse. 2019. Available from: https://sundhedsdatastyrelsen.dk/-/media/sds/ filer/find-tal-og-analyser/andre-analyser-og-rapporter/doedsaarsagsre gisteret/doedsaarsagsregisteret-2018.pdf?la=da. Accessed February 5, 2020.

17. Rustad P, Felding P, Franzson L, et al. The Nordic Reference Interval Project 2000: recommended reference intervals for 25 common biochemical properties. Scand J Clin Lab Invest. 2004;64(4):271-284. doi:10.1080/00365510410006324

18. Jepsen P, Vilstrup H, Andersen PK. The clinical course of cirrhosis: the importance of multistate models and competing risks analysis. Hepatology. 2015;62(1):292-302. doi:10.1002/hep.27598

19. Roshani D, Ghaderi E. Comparing smoothing techniques for fitting the nonlinear effect of covariate in Cox models. Acta Inform Med. 2016;24(1):38.
20. Hurvich CM, Tsai CL. Regression and time series model selection in small samples. Biometrika. 1989;76(2):297-307. doi:10.1093/biomet/ 76.2.297

21. Bernstorff M, Deichgræber P, Bruun NH, Dalsgaard E-M, Fengergrøn M, Lauritzen T. A randomised trial examining cardiovascular morbidity and all-cause mortality 24 years following general health checks: the Ebeltoft Health Promotion Project (EHPP). BMJ Open. 2019;9(10):e030400.

22. Christensen PB, Hay G, Jepsen P, et al. Hepatitis C prevalence in Denmark -an estimate based on multiple national registers. $B M C$ Infect Dis. 2012;12(1):178. doi:10.1186/1471-2334-12-178

23. Hansen N, Hay G, Cowan S, et al. Hepatitis B prevalence in Denmark - an estimate based on nationwide registers and a national screening programme, as on 31 December 2007. Eurosurveillance. 2013;18(47):20637. doi:10.2807/1560-7917.ES20 13.18.47.20637

24. Yamazaki H, Kamitani T, Matsui T, Yamamoto Y, Fukuhara S. Association of low alanine aminotransferase with loss of independence or death: a 5-year population-based cohort study. $J$ Gastroenterol Hepatol. 2019;34(10):1793-1799. doi:10.1111/jgh.v34.10
Clinical Epidemiology

\section{Publish your work in this journal}

Clinical Epidemiology is an international, peer-reviewed, open access, online journal focusing on disease and drug epidemiology, identification of risk factors and screening procedures to develop optimal preventative initiatives and programs. Specific topics include: diagnosis, prognosis, treatment, screening, prevention, risk factor modification,

Submit your manuscript here: https://www.dovepress.com/clinical-epidemiology-journal

\section{Dovepress}

systematic reviews, risk \& safety of medical interventions, epidemiology \& biostatistical methods, and evaluation of guidelines, translational medicine, health policies \& economic evaluations. The manuscript management system is completely online and includes a very quick and fair peer-review system, which is all easy to use. 